ACTA MYCOLOGICA

Vol. 49 (1): 109-134

2014
Dedicated to Professor Maria Ławrynowicz on the occasion of the 45th anniversary of her scientific activity

DOI: $10.5586 / \mathrm{am} .2014 .011$

\title{
Annotated checklist of fungi in Cyprus Island. 1. Larger Basidiomycota
}

\author{
MIGUEL TORREJÓN \\ La Estrella, 18-1, E-12410, Altura \\ torrejon.miguel@gmail.com
}

Torrejón M.: Annotated checklist of fungi in Cyprus Island. 1. Larger Basidiomycota. Acta Mycol. 49 (1): 109-134, 2014.

An annotated checklist of wild fungi living in Cyprus Island has been compiled broughting together all the information collected from the different works dealing with fungi in this area throughout the three centuries of mycology in Cyprus. This part contains 363 taxa of macroscopic Basidiomycota.

Key words: macromycetes, inventory, ecology, distribution

\section{INTRODUCTION}

In the first record, Chatin (1897) dealt with Terfezia aphroditis. Nattrass (1937) in the most important work ever done in Cyprus, were documented 92 species of anamorphic fungi, 104 species of ascomycetes, 148 species of basidiomycetes and 22 species of zygomycetes; Nattrass \& Papaioannou (1938) added 20 species more. The same year Dearness (1938), in a note, dealt with the species from the work of Nattrass (1937), but there were no news. Altson (1956), in a research into the cause of a rootrot illness on Vicia faba, contributed with one species. The following year, 44 samples more were studied in Georghiou \& Papadopoullos (1957). Sixteen years later, all the previous information was compiled in the work of Zyngas (1973) in which, 102 new references to the island were reported by the author. Ten years later Willoughby (1983) dealt with Rhizophlyctis rosea. In the new millennium, Winey (2005) in an illustrated book about macromycetes described 10 ascomycetes and 180 basidiomycetes to the island. In the next years, most authors dealt with single species originating from Cyprus: Tsopelas \& Nicolau (2005) reported Heterobasidion annosum for the first time and Tsopelas et al. (2008) researched Seiridium cardinale and Neophytou \& Ioannou (2009) recorded Graphiola phoenicis. Loizides (2008) dealt with macrofungi: five species of ascomycetes and twenty-seven basidiomycetes. Momany \& Gücel (2009) in an illustrated guide gave information about 105 different species 
of macromycetes. The same year in Momany et al. (2009) nine species of Agaricus were reported, but there is no new information in relation to the preceding work. Also, Edible and Toxic Fungi of Cyprus, by Loizides et al. (2011) is an important contribution improving the knowledge of macromycetes, in which eleven species of ascomycetes and eighty eight species of basidiomycetes were treated. Besides, Loizides (2008), Loizides \& Kyriakou (2011) and Loizides et al. (2012) concentrated on macromycetes. Finally, Torrejón (2013) added 25 species of anamorphic fungi, 28 species of ascomycetes, and 26 species of basidiomycetes to the island as you can see in Table 1.

Table 1

Published data on fungi in Cyprus Island

\begin{tabular}{|l|c|c|c|c|}
\hline $\begin{array}{c}\text { Authors' names of the works dealing } \\
\text { with fungi in Cyprus }\end{array}$ & $\begin{array}{c}\text { Number of } \\
\text { anamorphic }\end{array}$ & $\begin{array}{c}\text { Number of } \\
\text { ascomycetes }\end{array}$ & $\begin{array}{c}\text { Number of } \\
\text { basidiomy- } \\
\text { cetes }\end{array}$ & $\begin{array}{c}\text { Number of } \\
\text { zygomycetes }\end{array}$ \\
\hline Chatin (1897) & & 1 & & \\
\hline Nattrass (1937) & 92 & 104 & 148 & 22 \\
\hline Nattrass \& Papaioannou (1938) & 2 & 5 & 8 & 5 \\
\hline Altson (1956) & 1 & & & 2 \\
\hline Georghiou \& Papadopoullos (1957) & 4 & 14 & 24 & 10 \\
\hline Zyngas (1973) & 26 & 62 & 4 & \\
\hline Willoughby (1983) & 1 & & & \\
\hline Winey (2005) & & 10 & 180 & \\
\hline Tspelas \& Nicolau (2005) & & 1 & & \\
\hline Tspelas et al. (2008) & & 5 & 27 & \\
\hline Loizides (2008) & & 2 & 1 & \\
\hline Neophytou \& Ioannou (2009) & & 11 & 103 & \\
\hline Momany \& Gücel (2009) & & 4 & & \\
\hline Loizides et al. (2011) & & 28 & 26 & \\
\hline Loizides \& Kyriakou (2011) & & $\mathbf{2 4 8}$ & $\mathbf{6 2 4}$ & $\mathbf{3 9}$ \\
\hline Loizides et al. (2012) & $\mathbf{1 5 1}$ & & & \\
\hline Torrejón (2013) & & & \\
\hline Total no. species & & & & \\
\hline
\end{tabular}

\section{MATERIALS AND METHODS}

All the previous information dealing with fungi in Cyprus Island were compiled. The different collections were disposed chronologically in each single species including the name of the species and the authors' epithets, substrate/habitat, references and notes if necessary. Duplicated species from two or more authors were grouped in a single one.

\section{RESULTS AND DISCUSSION}

138 species of anamorphs, 195 species of ascomycetes, 543 species of basidomycetes and 33 species of zygomycetes have been arranged alphabetically in chronological order. For further information see Table 2. 
Table 2

Numbers of fungal species listed in Cyprus Island

\begin{tabular}{|l|c|c|c|c|}
\hline & $\begin{array}{c}\text { Number of } \\
\text { anamorphic }\end{array}$ & $\begin{array}{c}\text { Number of } \\
\text { ascomycetes }\end{array}$ & $\begin{array}{c}\text { Number of ba- } \\
\text { sidiomycetes }\end{array}$ & $\begin{array}{c}\text { Number of } \\
\text { zygomycetes }\end{array}$ \\
\hline $\begin{array}{l}\text { Total species from Authors' } \\
\text { works dealing with fungi in } \\
\text { Cyprus }\end{array}$ & $\mathbf{1 5 1}$ & $\mathbf{2 4 8}$ & $\mathbf{6 2 4}$ & $\mathbf{3 9}$ \\
\hline $\begin{array}{l}\text { Number of the same species } \\
\text { from two or more works }\end{array}$ & $\mathbf{1 3}$ & $\mathbf{5 3}$ & $\mathbf{8 1}$ & $\mathbf{6}$ \\
\hline Total species in Cyprus island & $\mathbf{1 3 8}$ & $\mathbf{1 9 5}$ & $\mathbf{5 4 3}$ & $\mathbf{3 3}$ \\
\hline
\end{tabular}

The summarized list contains 909 taxa and this causes the necessity of publishing the major part in the next issue(s). The present part contains the survey of larger Basidiomycota (363 taxa).

Species have been arranged alphabetically and Latin names and authors' epithets according to Index Fungorum.

Agaricus aridicola Geml, Geiser \& Royse [=Gyrophragmium dunalii (Fr.) Zeller] On dunes, Girne, Jan. 2005.Wynei (2005).

Agaricus augustus Fr.

In black and calabrian pine forest, early autumn. Loizides et al. (2008, 2011). Uncommon.

Agaricus bernardii Quél.

On dunes and roadsides in land, Alevkaya, autumn and winter. Momany \& Gücel (2009).

Agaricus bisporus (J.E. Lange) Imbach

In pastures, roadsides and cypress groves, autumn and winter. Loizides et al. (2011). Locally abundant.

Agaricus bitorquis (Quél.) Sacc.

Found in a stone quarry path, Beşparmark, Mar. 2000. In gardens, fields, parks and roadsides, autumn. Wynei (2005); Loizides et al. (2011). Rare.

Agaricus bresadolanus Bohus

Among grass in woodland or gardens, Alevkaya, Mar. Momany \& Gücel (2009).

Agaricus campestris L.

On grassy fields and newly dug garden soil, Karaman, Feb. 1997. On litter and pasture, Alevkaya, winter and spring. In filds, pastures and cultivated land, winter and spring. Wynei (2005); Loizides (2008); Momany \& Gücel (2009); Loizides et al. (2011). Frequent, but its numbers seem to be diminishing, probably due to the loss of its habitat.

Agaricus cappellianus Hlaváček (as Agaricus vaporarius (Vittad.) M. M. Moser)

On pasture and lawns and in deciduous woods, Alevkaya, autumn. Momany \& Gücel (2009).

Agaricus gennadii (Chatin \& Boud.) P. D. Orton

Under Eucalyptus sp., Köprülü reservoir, Dec. 2000. Wynei (2005).

Agaricus impudicus (Rea) Pilát

In all kind of forests, autumn. Loizides et al. (2011). Common.

Agaricus iodosmus Heinem.

In wood edges, parks and gardens, early in the autumn. Loizides et al. (2011). Rare. 
Agaricus moelleri Wasser

In all kind of forests, autumn and winter. Loizides et al. (2011). Occasional.

Agaricus placomyces Peck

On pastures and in mixed woodland on calcareous soils, Alevkaya, winter. Momany \& Gücel (2009).

Agaricus porphyrizon P. D. Orton

At the edge of a pinewood, Beşparmak, Dec. 2001. Wynei (2005).

Agaricus porphyrocephalus $\mathrm{F}$. H. MØller

On pastures and in mixed woodland, Alevkaya, winter. Momany \& Gücel (2009).

Agaricus pseudopratensis (Bohus) Waser

In filds, roadsides, wood edges and cypress groves, autumn and winter. Loizides et al. (2011). Frequent.

Agaricus spp.

Without data. Loizides (2008).

Agaricus sylvaticus Schaeff.

In mixed forests under pine trees, Alevkaya, autumn. In all kinds of forests, autumn and spring. Momany \& Gücel (2009).

Agaricus sylvicola (Vittad.) Peck.

Amongst grass in mixed forests, Alevkaya, autumn. Momany \& Gücel (2009); Loizides et al. (2011.)

Agaricus xanthodermus Genev.

In gardens, parks, wood edges, roadsides and cypress groves, between autumn and spring. Loizides et al. (2011). Appearing sparingly.

Agaricus aff. macrocarpus

On turf and pine litter, Yaila, Jan. 2001. Wynei 2005. The only reason for qualifying the name with "aff." is that the original description of the species indicates of coarse thick scales on the underside of the ring, which were no apparent in this collection.

Agaricus aff. sylvaticus

Without data. Wynei (2005).

Agrocybe cylindracea (DC.) Maire

On broadleaved logs and trunks (without date). Loizides et al. (2011). Rare.

Agrocybe praecox (Pers.) Fayod

Amongst grasses in mixed forests, Alevkaya, spring. Momany \& Gücel (2009).

Amanita cistetorum Contu \& Pacioni

It grows exclusively with Cistus, mainly $C$. salviifolius and $C$. monspeliensis, often very deeply rooted in the soil. Loizides \& Kyriakou (2011).

Amanita codinae (Maire) Bertault

On sandy coastal soil, the Cengizköy golf-course, Jan. 2001. Wynei (2005).

Amanita ovoidea (Bull.) Link

Under Pinus halepensis, Ktima, Dec. 1932. Under or near pines, Kalkanli, Nov. 1999. In black and calabrian pine forests, as well as aleppo oak stands, autumn and winter. Nattrass (1937); Wynei (2005). Loizides et al. (2011). As Amanita ovoidea Bull. in the first reference.

Amanita pantherina (DC.) Krombh.

In black pine forests, in early autumn. Loizides et al. (2011). Rare.

Amanita phalloides (Vaill. ex Fr.) Link

Under the bark of Populus nigra, Lefkara, Sep. 1933. Wynei (2005); Loizides (2008). 
Amanita proxima Dumée

On a gravelly roadside, Alevkaya, Nov. 1997. In black and calabrian pine forests, as well as aleppo oak stands, autumn and winter. Wynei (2005); Loizides et al. (2008, 2011).

Amanita torrendii Justo [=Torrendia pulchella Bres.]

Under Cistus as well as other mediterranean maquis (without date). Loizides \& Kyriakou (2011). Rare.

Amanita verna (Bull.) Lam.

Alevkaya forests, spring. Momany \& Gücel (2009).

Amanita virosa (Fr.) Bertill.

On soil surface in mixed or deciduous woodland, under a stone near Boğaz water pump, Apr. Loizides (2008); Momany \& Gücel (2009).

Amanita aff. malleata

Under Holly-leaved oak, Nov. 2000. Wynei (2005).

Ampulloclitocybe clavipes (Pers.) Redhead, Lutzoni, Moncalvo \& Vilgalys [=Clitocybe clavipes (Pers.) P. Kumm.]

In mixed forests especially with coniferous trees, Alevkaya, autumn. Momany \& Gücel (2009). Uncommon.

Antrodia serialis (Fr.) Donk

It spreads in vertical patches along the fissures of pine bark, Karaman, Dec. 1999. Wynei (2005).

Armillaria mellea (Vahl) P. Kumm. [=Armillariella mellea (Vahl) P. Karst.]

On roots and collar of Citrus sinensis, Famagusta; on Morus alba, Lapithos; on Salix alba, Kythrea. Without data. On Corylus avellana (without date and site). In a garden, growing from the underground stump of a falled tree, probly a plum, Karaman, Dec. 1999. On various broadleaved trees, autumn and winter. Nattrass (1937); Zyngas (1973 from Georghiou \& Papadopoulos 1957); Wynei (2005); Loizides et al. (2011). Frequent. As Armillariella mellea (Vahl) P. Karst. in the second reference.

Armillaria obscura (Schaeff.) Herink [=Armillaria polymyces (Pers.) Singer \& Clémençon]

Under Ficus trees, Alevkaya-Aldalya, spring. Momany \& Gücel (2009).

Astraeus hygrometricus (Pers.) Morgan

On soil surface in mixed forests, Alevkaya, Mar. In Cistus maquis, winter and spring. Momany \& Gücel (2009); Loizides \& Kyriakou (2011).

Auricularia auricula-judae (Bull.) Quél.

On dead wood of Citrus limonium, Kythrea, Oct. 1933; Famagusta, Oct. 1933; On dead wood of Juglans regia, Ktima, Oct. 1933. On a falled mulberry, Karaman, Feb. 1998. On branches and logs of various broadleaved trees, from autumn to spring. Nattrass (1937); Wynei (2005); Loizides et al. (2011). In the first references as $A u$ ricularia auricula-judae (Linn.) Schröt. Common.

Battarrea guicciardiniana Ces.

Under Eucalyptus sp., Nicosia, Nov. 1933. Nattrass (1937).

Battarrea phalloides (Dicks.) Pers.

Amongst lawns and under tree forests, Alevkaya, spring. Momany \& Gücel (2009).

Battarrea stevenii (Libosch.) Fr.

Under olives, Beyköy, (without date). Wynei (2005). Abundant in this habitat. 
Bjerkandera adusta (Willd.) P. Karst.

On the concrete walls of a manhole, Iskele, (without date). Wynei (2005).

Boletus aereus Bull.

In black and calabrian pine forests, golden oak forests and mediterranean maquis, autumn. Loizides et al. (2011). Rare.

Boletus boudieri Quél.

Under Pinus halepensis, Kornos, Oct. 1931. Nattrass (1937).

Boletus legaliae Pilát

Under oak, early in autumn. Loizides et al. (2011).

Boletus reticulatus Schaeff. (as Boletus reticulatus Boud.)

In black and calabrian pine forests, autumn. Loizides et al. (2011). Rare.

Boletus rhodopurpureus Smotl.

In a coniferous area, under an isolated pair of broadleaved trees (carob and hollyleaved oak), Esentepe, Nov. 1997. Wynei (2005).

Boletus rhodoxanthus (Krombh.) Kallenb.

In black and calabrian pine forests, as well as golden oak maquis, autumn. Loizides et al. (2011). Common in some years.

Boletus subtomentosus L. [=Xerocomus subtomentosus (L.) Quél.]

In black and calabrian pine forests, autumn. Loizides et al. (2011).

Bovista nigrescens Pers.

On the turf at the golf course, Cengizköy, 2001. Wynei (2005).

Bovista plumbea Pers.

On the turf at the folg course, Cengizköy, 2001. On lawns amongst short grass and pasture, Karşiyaka village, Mar. Wynei (2005); Momany \& Gücel (2009).

Byssomerulius corium (Pers.) Parmasto

On cut wood and bark of carob, (without data). Wynei (2005).

Cantharellus cibarius Fr.

In pine forest, Alevkaya, autumn. Momany \& Gücel (2009).

Cantharellus subpruinosus Eyssart. \& Buyck

In black and calabrian pine forests, in autumn and winter. Loizides et al. (2011).

Chalciporus amarellus (Quél.) Bataille

Under pines, Alevkaya, Jan. 1998. Wynei (2005).

Chlorophyllum molybdites (G. Mey.) Massee

In gardens, parks, greenhouses, roadsides and fields. Loizides et al. (2011). Rare.

Chondrostereum purpureum (Pers.) Pouzar

Parasitic or saprotrophic on many trees, Alevkaya, in the whole year. Momany \& Gücel (2009).

Chroogomphus rutilus (Schaeff.) O. K. Mill.

Under pines, growing on short turf, Alevkaya, Jan. 1998. In pine forest, Alevkaya, autumn. In black and calabrian pine forests, from autumn to spring. Wynei (2005); Momany \& Gücel (2009); Loizides et al. (2011). Common.

Clathrus ruber P. Micheli ex Pers.

Under Quercus infectoria, Platres, Jun. 1931. Under Cistus creticus, Alevkaya, spring.

Nattrass (1937); Momany \& Gücel (2009).

Clavaria incarnata Weinm.

Growing out of cypress debris, Yayla, Jan. 2001. Wynei (2005). 
Clavulina coralloides (L.) J. Schröt. [=Clavulina cristata (Holmsk.) J. Schröt.]

On pine litter, Alevkaya, Jan. 1998. Wynei (2005).

Cleistocybe carneogrisea (Malençon) Wizzini [=Hygrophorus carneogriseus Malençon] Only on cypress litter, Alevkaya, Jan. 2001. Wynei (2005).

Clitocybe alexandri (Gillet) Gillet

Under coniferous or broadleaved trees, Essentepe, Dec. 1998. In calabrian pine forests, in late autumn and spring. Wynei (2005); Loizides et al. (2011). Common.

Clitocybe augeana (Mont.) Sacc.

Growing on manure (without date). Wynei (2005).

Clitocybe candicans (Pers.) P. Kumm.

Girne, Feb. 2000 (without data of substrate). Wynei (2005).

Clitocybe costata Kühner \& Romagn.

Under pines, Alevkaya-Yayla, Dec. 2000. Wynei (2005).

Clitocybe diatreta (Fr.) P. Kumm.

Under pines, Esenteppe, Nov. 2000. Wynei (2005).

Clitocybe ditopa (Fr.) Gillet

Growing in a little cluster under pines, Alevkaya, Nov. 2000. Wynei (2005).

Clitocybe fragrans (With.) P. Kumm.

Amongst grasses, mosses or leaf litter beneath deciduous trees and pine forest, Alevkaya, Mar. Momany \& Gücel (2009).

Clitocybe houghtonii (W. Phillips) Dennis

On grass in mixed woodland forest, Alevkaya, Mar. Momany \& Gücel (2009).

Clitocybe obsoleta (Batsch) Quél.

Growing in "fairy rings" in moss under Cistus bushes, Alsancak, Dec. 2001. Wynei (2005).

Clitocybe odora (Bull.) P. Kumm.

In calabrian pine forests, alluvial broadleaved forests and aleppo oak stands, autumn and winter. Loizides et al. (2011). Occasional.

Clitocybe rivulosa (Pers.) P. Kumm.

In gardens, parks, roadsides and wood edges, autumn and winter. Loizides et al. (2011). Uncommon.

Clitocybe umbilicata (Schaeff.) P. Kumm.

On moss under pines, Greçitköy reserve, Dec. 2001. Wynei (2005).

Clitocybe vermicularis (Fr.) Quél.

On pine litter, Alevkaya, Mar. 1997. Wynei (2005).

Clitocybe spp.

Without data. Loizides (2008).

Clitopilus prunulus (Scop.) P. Kumm.

On grass near Eucalyptus, Kanliköy, Dec. 1998. Wynei (2005).

Coltricia perennis (L.) Murrill

Amongst grass on heath and woodland, Alevkaya, spring. Momany \& Gücel (2009). Conferticium ochraceum (Fr.) Hallenb.

On decaying wood of Pinus nigra subsp. palliata, Troodos, 17 Nov. 2011. Torrejón (2013).

Conocybe apala (Fr.) Arnolds [=Bolbitius tener (Gray) Berk.]

On mature-rich grass beside Eucalyptus sp., Kanliköy, Dec. 1998. Wynei (2005). 
Conocybe filaris (Fr.) Kühner [=Pholiotina filaris (Fr.) Singer]

On moss in a sandstone valley, Kalkanli, Feb. 2000. Wynei (2005).

Conocybe pulchella (Velen.) Hauskn. \& Svrček [=Conocybe pseudopilosella Kühner ex Kühner \& Romagn.]

Among grass and lawns in woodland, Alevkaya, spring. Momany \& Gücel (2009).

Conocybe subovalis Kühner \& Watling [=Galera ovalis (Fr.) Gillet]

On ground, Nicosia, (without data). Nattrass (1937).

Coprinellus aff. flocculosus [= Coprinus aff. flocculosus]

On the side of a tiny brook, Kalavaç, Apr. 2000. Wynei (2005).

Coprinellus domesticus (Bolton) Vilgalys, Hopple \& Jacq. Johnson $[=$ Coprinus domesticus (Bolton) Gray]

On buried timber, Nov. 1997. Wynei (2005).

Coprinopsis atramentaria (Bull.) Redhead, Vilgalys \& Moncalvo [=Coprinus atramentarius (Bull.) Fr.]

On ground, Nicosia, Mar. 1932. Nattrass (1937).

Coprinopsis cinerea (Schaeff.) Redhead, Vilgalys \& Moncalvo [=Coprinus cinereus (Schaeff.) Gray]

Growing in a large numbers mixed with another smaller fungus on a sheep midden. Wynei (2005).

Coprinopsis cothurnata (Godey) Redhead, Vilgalys \& Moncalvo [=Coprinus cothurnatus Godey]

In a midden-heap on a sheep dropping, Yeniceköy, Jan. 1998. Wynei (2005).

Coprinopsis picacea (Bull.) Redhead, Vilgalys \& Moncalvo [=Coprinus picaceus (Bull.) Gray]

Mixed woods, Alevkaya, Jan. 2000; Under Pistacia terebinthus, Küçükerenköy, Jan. 2000. On soil surface in mixed forests, Karşiyaka village, Apr. Wynei (2005); Momany \& Gücel (2009).

Coprinopsis romagnesiana (Singer) Redhead, Vilgalys \& Moncalvo

On or around rotten wood (without date and site). Loizides et al. (2011). Uncommon.

Coprinus comatus (O. F. Müll.) Pers.

On a rubbish dump, Esentepe, Dec. 2000; on ploughed soil, At Ağillar, Feb. 2001. Without data. In grass by roadsides, rubbish heaps or lawns and under trees on remnants of fallen leaves, Girne-Alsancak, Feb. In parks, fields, wood edges and road edges, often on disturbed ground, autumn and spring. Wynei (2005); Loizides et al. (2008, 2011); Momany \& Gücel (2009). As Coprinus ovatus (Schaeff.) Fr. in the second habitat from the first reference.

Coprinus disseminatus (Pers.) J. E. Lange

On stumps of broadleaved trees or on soil, Karaoğlanoğlu, Mar. Momany \& Gücel (2009).

Coprinus spp.

Without data. Loizides (2008).

Coriolellus albidus (Fr.) Bondartsev

On fallen or dead branches of deciduous trees, Alevkaya, May. Momany \& Gücel (2009). 
Coriolopsis gallica (Fr.) Ryvarden [=Trametes hispida Bagl.]

On branch of Prunus armeniaca, Ay. Amvrosios, Kyrenia, Jun.1931. On live walnut and on cut timber, (without site and date). Nattrass (1937); Wynei (2005).

Coriolus hirsutus (Wulfen) Quél.

On dead wood and fallen trunks of deciduous trees, Alevkaya, May. Momany \& Gücel (2009).

Cortinarius caligatus Malençon

In calabrian pine forests and mediterranean maquis in association with rockrose Cistus sp. In Cistus maquis, (without date). Loizides et al. (2011); Loizides \& Kyriakou (2011).

Cortinarius collinitus (Pers.) Fr.

On soil in mixed forests and thorny shrubs, more rarely in deciduous, Gecitköy, Mar. Momany \& Gücel (2009).

Cortinarius dionysae Rob. Henry

In Cistus garigue, Beşparmak, Dec. 2000. Wynei (2005).

Cortinarius juranus Remaux

In calabrian pine and golden oak forests, autumn and winter. Loizides et al. (2011). Rare.

Cortinarius mucosus (Bull.) J. Kickx

In conifer heath, usually with pine, Alevkaya, autumn. Momany \& Gücel (2009).

Cortinarius splendens Rob. Henry

In calabrian pine and golden oak forests, autumn and winter. Loizides et al. (2011).

Cortinarius sp.

On burn ground in Cistus and Lentisk garigue, Buffavento track, Jan. 2001. Wynei (2005).

Crepidotus cesatii (Rabenh.) Sacc.

On dead twigs of Dittrichia viscosa, Özhan, Dec. 1998. Wynei (2005).

Crepidotus mollis (Schaeff.) Staude

On a carob, Tazik Kiran woods, Dec. 1998. Wynei (2005).

Crepidotus variabilis (Pers.) P. Kumm.

On terebinth twigs, Jan. 1998. Wynei (2005).

Crinipellis scabella (Alb. \& Schwein.) Murrill

On dead grass stems, Gönyeli, Nov. 1997. Wynei (2005).

Cryptosporiopsis corticola (Edgerton) Nannf. [=Mysosporium corticolum Edgerton] On dead twigs and cankers on branch of Pyrus malus, Prodhromos, Oct. 1931. Nattrass (1937).

Cyathus olla (Batsch) Pers.

On a heap of plant remains, Kanliköy, Dec. 1998. Wynei (2005).

Cystoderma terreyi (Berk. \& Broome) Harmaja

Between Alevkaya and Yayla, Dec. 1998. Wynei (2005).

Cystoderna amianthinun (Scop.) Fayod

In pineland, Alevkaya, Jan. 1998. Wynei (2005). Frequent.

Cystolepiota adulterina (F. H. Møller) Bon [=Lepiota adulterina F. H. Møller]

Amongst broadleaved woods as nettle and scrub, Alevkaya, autumn. Momany \& Gücel (2009).

Cystolepiota cystophora (Malençon) Bon

On stony ground under carobs, Alevkaya, Ja. 1999. Wynei (2005). 
Cystolepiota moelleri Knudsen [=Lepiota rosea Rea]

On damp ground under deciduous trees, Alevkaya, autumn. Momany \& Gücel (2009).

Dacrymyces variisporus $\mathrm{McNaab}$

On wood from branches of Pinus nigra subsp. palliata, Troodos, 17 Nov. 2011. Torrejón (2013).

Dacryobolus karstenii (Bres.) Oberw. ex Parmasto [=Stereum karstenii Bres.]

On wood of Pinus halepensis, Troödos, Aug. 1931. Nattrass (1937).

Entoloma hirtipes (Schumach.) M. M. Moser [=Nolanea hirtipes (Schumach.) P. Kumm.]

On grass or on rich humus in pine forests, Alevkaya, Mar. Momany \& Gücel (2009).

Entoloma jubatum (Fr.) P. Karst.

In pinewoods, Alevkaya, Jan. 1998. Wynei (2005). Frequent.

Entoloma lividoalbum (Kühner \& Romagn.) Kubička

In calabrian pine and golden oak forests, autumn and winter. Loizides et al. (2011).

Occasional.

Entoloma phaeocyathus Noordel.

In the Cengizköy golf-course, Dec. 2001. Wynei (2005).

Entoloma aff. hirtipes

In pinewoods, Alevkaya (without date). Wynei (2005).

Entoloma aff. undatum

On mossy ground under pines, St. Hilarion, Dec. 1998. Wynei (2005).

Exidia plana Donk

On dead deciduous wood, Alevkaya, Dec. 1998. Wynei (2005).

Exidiopsis calcea (Pers.) K.Wells

On wood from a branch of Pinus brutia, Trail to Calydonian Falls, 19 Nov. 2011. Torrejón (2013).

Exobasidium vaccinii (Fuckel) Woronin

On leaves of Arbutus andrachne Troodos, Jun. 1935. Nattrass (1937).

Fomes fomentarius (L.) Fr.

On trunk of Populus nigra, Saitta, Feb. 1932; of Platanus orientalis, Stavros, Jan. 1936.

On birch and on almond, Alevkaya and Pedoulas, Mar. Nattrass (1937); Momany \& Gücel (2009).

Fomitiporia punctata (Pilát) Murrill [=Poria friesiana Bres.] [=Phellinus punctatus Pilat]

On Citrus sinensis, Famafusta, Jul. 1933. On olive stumps and roots, Karaman, (without date). Nattrass (1937); Wynei (2005).

Fuscoporia torulosa (Pers.) T. Wagner \& M. Fisch. [=Fomes torulosus (Pers.) Lloid] [=Phellinus torulosus (Pers.) Bourdot \& Galzin]

On trunk of Ceratonia siliqua, Lefkara, Apr. 1931. Attached to carob and almond trees, (without site and date). On tree stumps, carob tree stem, pomegranate, Kasyake, Lapta, Alevkaya, Mar. Nattrass (1937); Wynei (2005); Momany \& Gücel (2009).

Galerina marginata (Batsch) Kühner

Beside a grassy firebreak, Alevkaya, Jan. 2002. On dead (sometimes buried) wood, autumn and winter. Wynei (2005); Loizides et al. (2008, 2011). Frequent. 
Galerina pumila (Pers.) M. Lange [=Galerina mycenopsis (Fr.) Kühner]

On soil amongst moss in grassland, Tepebaşi altkisimlari, Mar. Momany \& Gücel (2009).

\section{Galerina vittiformis (Fr.) Singer}

On mossy soil among Cistus, under pines, Dec. 1999, (without site). Wynei (2005).

Ganoderma adspersum (Schulzer) Donk

Round a fig stem and on the buried remains of a dead almond, Karaman, (without date). Wynei (2005); Momany \& Gücel (2009).

Ganoderma applanatum (Pers.) Pat.

On trunk of Ceratonia siliqua, Ay. Theodhoros, Larnaca, Jun. 1931; on root of Alnus orientalis, Marathasa, Mar. 1936. On Citrus limonium, (without date and site). Nattrass (1937); Zyngas (1973 from Nattrass \& Papaioannou 1938).

Ganoderma lucidum (Curtis) P. Karst.

On ground, Agros, Oct. 1934. Nattrass (1937).

Ganoderma resinaceum Boud.

On stumps of carobs, Alevkaya, (without date). Momany \& Gücel (2009).

Ganoderma sp. (as Ganoderma solani (Mart.) Sacc.)

On stem of Ceratonia siliqua, Akanthou, 1967. Zyngas (1973).There is not a fungus with the name used by Zyngas. It might be Fuscoporia torulosa, the most common species living in this habitat in all mediterranean coastal area.

Geastrum berkeleyi Massee

On pine litter, Mt. Yayla, Dec. 2001. Wynei (2005).

Geastrum fimbriatum Fr. [=Geastrum sessile (Sowerby) Pouzar]

On rich humus or pine needles in mixed forest, Alevkaya, Mar. Momany \& Gücel (2009).

Geastrum rufescens Pers.

On soil surface in deciduous and coniferous woodland, Alevkaya, spring. Momany \& Gücel (2009).

Geastrum triplex Jungh.

On leaf litter in deciduous wood, parks and gardens and mainly under pine trees, Alevkaya, Feb. Momany \& Gücel (2009); Wynei (2005).

Geastrum pseudolimbatum Hollós

On a bare patch of sandy soil, Cengizköy golf course, Dec. 2011. Wynei (2005).

Gloeophyllum abietinum (Bull.) P. Karst. [=Lenzites abietina (Bull.) Fr.]

On worked timber, Nicosia, May 1931. On the sides and cut surfaces of falled cypresses and pines (without site and date). On wood tree stumps or durned forests, Alevkaya (without data). Nattrass (1937); Wynei (2005); Momany \& Gücel (2009). As Lenzites abietina (Bull.) Fr. In the first reference.

Gloeophyllum trabeum (Pers.) Murrill [=Lenzites trabea (Pers.) Fr.]

On trunk of Pinus halepensis, Stavros, Sep. 1935. On dead conifers and sawn wood, (without site and data). Nattrass (1937); Wynei (2005).

Grifola frondosa (Dicks.) Gray

On an indeterminate tree, Yenierenköy, (without date). Wynei (2005).

Gymnopilus flavus (Bres.) Singer

On soil with wood chippings, near Cengizköy paper mill, Dec. 2001. Wynei (2005).

Gymnopilus junonius (Fr.) P.D. Orton

On dead wood, autumn and winter. Loizides et al. (2011). Rare. 
Gymnopus dryophilus (Bull.) Murrill [=Collybia dryophila (Bull.) P. Kumm.]

On moss or grass under trees, from Oct., (without site). Wynei (2005).

Gymnopus peronatus (Bolton) Gray [=Collybia peronata (Bolton) P. Kumm.]

On leaf litter in mixed woodland forests, Alevkaya, spring. Momany \& Gücel (2009).

Hebeloma cistophilum Maire

Beneath Cistus salvifolius, C. albidus, C. monspeliensis and C. laurifolius, autumn and winter. Loizides \& Kyriakou (2011).

Hebeloma sinapizans (Paulet) Gillet (as Hebeloma sinapizans (Pers.) Fr.)

In black and calabrian pine forests as well as golden oak forests, autumn and winter. Loizides et al. (2011). Common.

Heterobasidion annosum (Fr.) Bref.

On stumps of Pinus nigra ssp. pallasiana,Troodos, Jun. 2003. Tsopelas \& Nikolaou (2005).

Hohembuehelia geogenia (DC. ex Fr.) Singer

On buried wood from olive debris, Alevkaya, Jan. 2001. Wynei (2005).

Hydnum rufescens Pers.

In black and calabrian pine forests, golden oak forests and alluvial broadleaved forests. Loizides et al. (2011). Rare.

Hygrocybe acutoconica (Clemen.) Singer [=Hygrocybe langei Kühner]

Amongs lawns and pasture, Alevkaya, autumn. Momany \& Gücel (2009).

Hygrocybe chlorophana (Fr.) Wünsche

On grassy patches, Esentepe and Bahçeli, 1999. Wynei (2005).

Hygrocybe conica (Schaeff.) P. Kumm.

On lawn, Alevkaya, Feb. 1997; Lefkoça, Jan. 2001. Wynei (2005).

Hygrocybe virginea (Wulfen) P.D. Orton \& Watling [=Cuphophyllus virgineus (Wulfen) Kovalenko]

In shady places under pines, Esentepe, (without date). Wynei (2005).

Hygrophorus chrysodon (Batsch) Fr.

In Cistus maquis, (without date). Loizides \& Kyriakou (2011).

Hygrophorus cossus (Sowerby) Fr.

Amongst grass in mixed forests, Alevkaya, autumn. Momany \& Gücel (2009).

Hygrophorus hypothejus (Fr.) Fr.

In calabrian pine forests, winter. Loizides et al. (2011). Common.

Hygrophorus latitabundus Britzelm.

In a pinewood, Esentepe, Dec. 2000. In black pine forests, autumn. Wynei (2005); Loizides et al. (2011). Locally abundant, but not very widespread.

Hygrophorus pseudodiscoideus var. cistophilus Bon \& G. Riousset

Under Cistus salviifolius and C. monspeliensis, (without date). Loizides \& Kyriakou (2011).

Hygrophorus pustulatus (Pers.) Fr.

Amongst grass in mixed forest especially with pine, Alevkaya, spring. Momany \& Gücel (2009).

Hygrophorus spp.

Without data. Loizides (2008).

Hyphoderma incrustatum K. H. Larss.

On decaying wood from a log of Pinus nigra subsp. palliata, Troodos, 17 Nov. 2011. Torrejón (2013). 
Hyphoderma nemorale K. H. Larss.

On decaying wood from a branch of Quercus alnifolia, Trail to Calydonian Falls, 19 Nov. 2011.Torrejón (2013).

Hyphodontia sambuci (Pers.) J. Erikss.

On dead deciduous wood, Alevkaya, Mar. Momany \& Gücel (2009).

Hypholoma capnoides (Fr.) P. Kumm.

On stumps of unknown trees, Alevkaya, spring. Momany \& Gücel (2009).

Hypholoma fasciculare (Huds.) P. Kumm.

On dead or buried wood, autumn and spring. Loizides et al. (2011).

Hypholoma marginatum J. Schröt.

On soil under conifers, Karaoğlanoğlu, Mar. Momany \& Gücel (2009).

Infundibulicybe geotropa (Bull.) Harmaja

In calabrian pine forest, aleppo oak stands and alluvial broadleaved forests, autumn and winter. Loizides et al. (2011).

Inocybe bongardii var. pisciodora (Donadini \& Riousset) Kuyper [=Inocybe pisciodora Donadini \& Riousset]

On turf under Cistus, Tarzik Viran, Feb. 2000. Wynei (2005). Numerous in this habitat.

Inocybe cincinnata (Fr.) Quél.

Amongst grass in deciduous and coniferous woodlands, Alevkaya, spring. Momany \& Gücel (2009).

Inocybe dulcamara (Pers.) P. Kumm.

Small troops on turf under Cistus and at wood edges, Karaman, May 2000. On dunes or lawns, Alevkaya, winter.

Wynei (2005); Momany \& Gücel (2009).

Inocybe flocculosa (Berk.) Sacc. [=Inocybe flocculosa var. crocifolia (Herink) Kuiper] On a sandstone valley soil, under Cistus and Inula shrublets, Kalkanli, Feb. 2000; under pines, Alevkaya, Jan. 2002. On soil surface in mixed forests, Akdeniz Drmanlari, Mar. Inside or outside forests, often on calcareous soil, autum, and winter and spring. In Cistus maquis, (without date). Wynei (2005); Momany \& Gücel (2009); Loizides et al. (2011); Loizides \& Kyriakou (2011). Common. As Inocybe flocculosa var. crocifolia in the first reference.

Inocybe fraudans (Britzelm.) Sacc.

In black and calabrian pine forests, autumn and winter. Loizides et al. (2011).

Inocybe geophylla (Fr.) P. Kumm.

In black and calabrian pine forests, cedar forests and alluvial broadleaved forests, autumn, winter and occasionally in spring. Loizides et al. (2011). Common.

Inocybe godeyi Gillet

In deciduous woodland and mixed forests, Alevkaya, autumn and spring. Momany \& Gücel (2009).

Inocybe griseolilacina J. E. Lange

Deciduous trees and roadsides, Alevkaya, winter. Momany \& Gücel (2009).

Inocybe griseovelata Kühner

In pinewoods, Esentepe, Dec. 1999. Wynei (2005).

Inocybe hirtella Bres.

In parks and deciduous forests, particularly under beech and lime trees on calcareous humus-rich soils, Alevkaya, Mar. Momany \& Gücel (2009). 
Inocybe lacera (Fr.) P. Kumm.

In pine woods, Alevkaya, Feb. 1998. Under pine trees, Alevkaya, winter. Wynei (2005); Momany \& Gücel (2009).

Inocybe obscurobadia (J. Favre) Grund \& D. E. Stuntz

Under pines, Anthos, Camlibel, Jan. 2000. Wynei (2005).

Inocybe petiginosa (Fr.) Gillet

Deciduous woodland, Tepebasi-Altkisimlari, Mar. Momany \& Gücel (2009).

Inocybe rimosa (Bull.) P. Kumm.

Mainly in black pine forests, less often in calabrian pine forests, early in the autumn.

Loizides et al. (2011). Very common.

Inocybe aff. bongardii

On pine litter, Alevkaya, Feb. 1999. Wynei (2005).

Inocybe aff. nitidiuscula

On moss under pines, Alevkaya, Feb. 1997. Wynei (2005).

Inocybe aff. sindonia

On pine litter, Alevkaya, Jan. 2002. Wynei (2005).

Inocybe spp.

Without data. Loizides (2008).

Inonotus hispidus (Bull.) P. Karst. [=Polyporus hispidus (Bull.) Fr.]

On trunk of Populus nigra, Nicosia, Nov. 1931. Nattrass (1937).

Lacrymaria lacrymabunda (Bull.) Pat. [=Psathyrella lacrymabunda (Bull.) M. M. Moser]

On soil and pine debris in a shaded courtyard, Karaman, Dec. 2000. Wynei (2005).

Lactarius chrysorrheus Fr.

Under golden oak, autumn and winter. Loizides et al. (2011). Common.

Lactarius deliciosus (L.) Gray

Under Pinus halepensis, Troodos, (without date). Under pines and among terevinth shrubs, between Alevkaya and the Bessparmak hills, (without date). Amongst grass under pines or spruce trees, Alevkaya, autumn and spring. In black and calabrian pine forests, autumn and winter. Nattrass (1937); Wynei (2005); Loizides (2008); Loizides et al. (2011); Momany \& Gücel (2009). As Lactarius deliciosus (Linn.) Fr. in the first reference. Frequent.

Lactarius hepaticus Plowr.

On litter in mixed forests especially with pine, Alevkaya, spring. Momany \& Gücel (2009).

Lactarius hysginus (Fr.) Fr.

In mixed forests, Alevkaya, winter. Momany \& Gücel (2009).

Lactarius mammosus Fr.

In mixed forests especially under pine trees, Gecitköy, Mar. Momany \& Gücel (2009).

Lactarius sanguifluus (Paulet) Fr.

Mainly in black pine forests in altitudes over $1.200 \mathrm{~m}$. Loizides et al. (2011). Common.

Lactarius semisanguifluus R. Heim \& Leclair

In calabrian pine forests, often in calcareous soil, autumn and winter. Loizides et al. (2011). Common. 


\section{Lactarius tesquorum Malençon}

In symbiosis with rockrose Cistus spp., autumn, winter and spring. In Cistus maquis, (without date). Loizides et al. (2011); Loizides \& Kyriakou (2011). Common. One of the commonest Cistus-specific species.

Lactarius volemus (Fr.) Fr.

Under trees in mixed forests, Alevkaya, winter. Momany \& Gücel (2009).

Lactarius spp.

Without data. Loizides (2008).

Laetiporus sulfureus (Bull.) Murrill [=Polyporus sulphureus (Bull.) Fr.]

On Ceratonia siliqua (without date and site). Kicked down on Melia azedarach, Girne, Nov. 1999. On dead or alive broadleaved trees, autumn and spring. Zyngas (1973 from Nattrass \& Papaioannou 1938); Wynei (2005); Loizides et al. (2011). As Polyporus sulphureus (Bull.) Fr. in the first reference.

Leccinellum corsicum (Rolland) Bresinsky \& Manfr. Binder

Associated with Cistus monspeliensis, C. ladanifer, C. incanus and C. salviifolius, (without date). Loizides \& Kyriakou (2011).

Leccinellum lepidum (H. Bouchet ex Essette) Bresinsky \& Manfr. Binder

Under golden oak and Kermes oak, autumn and winter. Loizides et al. (2011). Frequent in some years.

Lepiota aspera (Pers.) Quél.

In alluvial, broadleaved forests, parks, scrubland and roadsides, autumn. Loizides et al. (2011). Uncommon.

Lepiota brunneoincarnata Chodat \& C. Martín

Without data. In forests, parks, fields, roadsides and gardens, autumn and winter. Loizides (2008); Loizides et al. (2011). Occasional.

Lepiota castanea Quél.

In calabrian pine forests, alluvial broadleaved forests, wood edges and scrubland, autumn and winter. Wynei (2005); Loizides et al. (2011). Frequent.

Lepiota pseudolilacea Huijsman

In calabrian pine forests, roadsides, cypress groves, wood edges and scrubland, autumn and winter. Loizides et al. (2011). Occasional.

Lepiota subincarnata J. E. Lange

In calabrian pine forests, alluvial broadleaved forests, wood edges, paarks and gardens, often among grass, autumn and winter. Loizides et al. (2011). Occasional.

Lepiota aff. bruneoincarnata

On grassy bank, Gecitköy, Dec. 2001. Wynei (2005).

Lepiota aff. clypeolaria

On mossy turf, Alevkaya, Jan. 2002.Wynei (2005).

Lepista flaccida (Sowerby) Pat. [=Clitocybe inversa (Scop.) Quél.]

On moss under pines, Geçitköy reserve, Dec. 2001. Wynei (2005).

Lepista nuda (Bull.) Cooke

Around Girne and Alevkaya, Jan. and Feb. (without year). In all kind of forests, autumn and winter. Wynei (2005); Loizides (2008); Loizides et al. (2011).Quite frequent.

Lepista sordida (Schumach.) Singer

Without data. Loizides (2008). 
Leucoagaricus leucothites (Vittad.) Wasser

Numerous on turf, Buffavento road, Nov. 2000. In parks, gardens, road edges and round-about among grass, autumn and winter. Wynei (2005); Loizides et al. (2011). Leucoagaricus serenus (Fr.) Bon \& Boiffard [=Sericeomyces serenus (Fr.) Heinem.] Under pines, Cengizköy, Nov. 1998. Wynei (2005).

Limacella illinita (Fr.) Maire

In pine woods, St. Hilarion, Dec. 2000. Wynei (2005).

Limacella subfurfuracea Contu

In pinewoods, Alevkaya and Akdeniz, Dec. 1998, 2000. Wynei (2005).

Lycoperdon excipuliforme (Scop.) Pers.

In black and calabrian pine forests, as well as golden oak maquis, autumn and spring. Loizides et al. (2011). Frequent.

Lycoperdon nigrescens Pers. [=Lycoperdon foetidum Bonord.]

On soil in coniferous or mixed woodland, Alevkaya, Mar. Momany \& Gücel (2009). Lycoperdon perlatum Pers.

On soil under pines, Alevkaya (without date). On soil surface in mixed woodland, Alevkaya and Prodromos forests, Mar. In black and calabrian pine forests, autumn. Wynei (2005); Momany \& Gücel (2009); Loizides et al. (2011). Common.

Lycoperdon pratense Pers. [=Vascellum pratense (Pers.) Kreisel]

On turf, Dengizköy golf course, Dec. 2001. On soil surface in lawns and pasture, Karşiyaka village, Apr. Wynei (2005); Momany \& Gücel (2009).

Lycoperdon pyriforme Schaeff.

On rotten logs or stumps or on soil surface but attached to buried wood by a mycelial cords, Karşiyaka village, Mar. Momany \& Gücel (2009).

Lyophyllum connatum (Schumach.) Singer

In black and calabrian pine and golden oak forests. Loizides et al. (2011). Occasional.

Lyophyllum decastes (Fr.) Singer

In a garrigue, Buffavento dirt road, Jan. 2001. Wynei (2005).

Lyophyllum fumosum (Pers.) P. D. Orton

In black and calabrian pine and golden oak forests, autumn and winter. Loizides et al. (2011). Frequent in some years.

Lyophyllum sp. On the Cengizköy golf course, Dec. 2001. Wynei (2005).

Macrolepiota excoriata (Schaeff.) Wasser

In wood edges, fields, gardens and road edges, autumn and winter. Loizides et al. (2011).

Macrolepiota konradii (Huijsman ex P. D. Orton) M. M. Moser

In a pinewood, Alevkaya, Nov. 2000. Wynei (2005).

Marasmiellus ramealis (Bull.) Singer [=Marasmius ramealis (Bull.) Fr.]

On old wood material, Tepebasi-Altkisimlari, Mar. Momany \& Gücel (2009).

Marasmius anomalus Peck

On turf, Gönyeli, Jan. 1998. Wynei (2005).

Marasmius rotula (Scop.) Fr.

On turf, Armenian monastery, Nov. 2000. Wynei (2005).

Marasmius wynneae Berk. \& Broome (as Marasmius wynnei)

Under pines, Alevkaya, Dec. 1998. Wynei (2005). 
Melanoleuca brevipes (Bull.) Pat.

On floor of gravel quarry, Ridge road, (without date). Wynei (2005).

Melanoleuca excissa (Fr.) Singer

Amongs clover on sandy soil, Cengizköy golf course, Dec. 2004. Wynei (2005).

Melanoleuca paedida (Fr.) Künher \& Maire

On moss under Cistus bushes, Arapköy, Feb. 2001. Wynei (2005).

Melanoleuca rasilis var. rasilis (Fr.) Singer

Alongside a broken log on dunes, Girne, Dec. 2004. Wynei (2005).

Melanoleuca strictipes (P. Karst.) Jul. Schäff.

In woodland amongst grass or pasture, Alevkaya, autumn. Momany \& Gücel (2009).

Melanoleuca aff. excissa

On bare soil, Alevkaya, Feb. 2000. Wynei (2005).

Micromphale brassicolens (Romafn.) P. D. Orton

On pine-litter, Alevkaya, Nov. 2000. Wynei (2005).

Montagnea haussknechtii Rabenh.

On sand dunes, Karpaz, May 1997. Wynei (2005).

Mycena arcangeliana Bres. [= Mycena oortiana Hora]

On a dead mossy carob, Tazik Kiran, Feb. 1999. On tree stumps and branches of deciduous trees, Alevkaya, winter. Wynei (2005); Momany \& Gücel (2009).

Mycena amicta (Fr.) Quél.

Widespread on pine cones, Küçükerenköy, Jan. 2000. Wynei (2005).

Mycena crocata (Schrad.) P. Kumm.

On leaf litter in mixed forests, Beşparmak, winter. Momany \& Gücel (2009).

Mycena galericulata (Scop.) Gray

On fallen branches and stumps of broadleaved trees, Alevkaya, autumn. Momany \& Gücel (2009).

Mycena galopus (Pers.) P. Kumm.

On Cistus debris and pine litter, (without site and date). Amongst leaf litter in woodlands, Alevkaya, spring. Wynei (2005); Momany \& Gücel (2009). Fairly common.

Mycena megaspora Kauffman [=Mycena uracea A. Pearson]

On heath in grassland and mixed forests, Akdeniz Ormanlari, Mar. Momany \& Gücel (2009).

Mycena pelianthina (Fr.) Quél.

On litter in mixed forests, Alevkaya, spring.Momany \& Gücel (2009).

Mycena pura (Pers.) P. Kumm.

In pine woods, Kozan, Dec. 1998. In black, calabrian pines and cedar forests, as well as in alluvial broadleaved forests, autumn. Wynei (2005). Loizides et al. (2011). Common.

Mycena sanguinolenta (Alb. \& Schwein.) P. Kumm.

Growing from a cracked carob stump, Alevkaya, Dec. 2001. Wynei (2005). It was observed on soil and truf too.

Mycena seynesii Quél.

On pine cones on the ground or buried, Dec.-Jan. (without site). On pine cones and coniferous debris, Alevkaya, winter. Wynei (2005); Momany \& Gücel (2009). Common in pinewoods.

Mycena spp.

Without data. Loizides (2008). 
Myriostoma coliforme (Dicks.) Corda

Two basidiomata, on soil very rich in humus beneath Quercus alnifolia and Platanus orientalis, close to Cyclamen cyprius, Cedar Valley, 22 Nov. 2011. Torrejón (2013). Rare. Included by the European Council for the Conservation of Fungi as a candidate to the list of threatened fungi in Europe.

Myxomphalia maura (Fr.) Hora

Often in troops on burnt ground, as in garrigue, Buffavento dirt road, Jan. 2001. Wynei (2005).

Omphallina sp.

On a mossy boulder, Karaman, Dec. 1999. Wynei (2005).

Omphaltus olearius (DC.) Singer

On olive trees, (without date and site). On olive and other broadleaved trees, sometimes at the base of trunks appearing terrestrial, but always associated with wood, (without date). Wynei (2005); Loizides (2008); Loizides et al. (2011).

Panaeolina foenisecii (Pers.) Maire

In grassland and on loans and roadsides, Tepebasi altkisimlari, Mar. Momany \& Gücel (2009).

Panaeolus aff. olivaceus

On burnt ground, Karaman, Dec. 1999. Wynei (2005). This collection almost matches the descriptions of this rare European species which has somewhat broarder spores; hence the qualification "aff.".

Panaeolus papilionaceus (Bull.) Quél.

On a wayside grass, Atifonitis Monastery, Dec. 2000. Wynei (2005).

Panus conchatus (Bull.) Fr. [=Lentinus conchatus (Bull.) J. Schröt]

On soil between rocks, but perhaps attached to olive or carob roots, Karşiyaka, Jan. 2001. Wynei (2005).

Parasola megasperma (P. D. Orton) Redhead, Vilgalys \& Hopple [=Coprinus megaspermus P. D. Orton]

On the ground, Lapta, Dec. 2004. Wynei (2005).

Parasola plicatilis (Curtis) Redhead, Vilgalys \& Hopple [=Coprinus plicatilis (Curtis) Fr.]

On turf and shady wood sides, Alevkaya, Jan. 2001. Wynei (2005).

Paxillus rubicundulus P.D. Orton

In mycorrhizal symbiosis with alder, autumn and spring. Loizides et al. (2011).

Peniophora cinerea (Pers.) Cooke

On a decaying branch of Ceratonia siliqua, Asgata I, 24 Nov. 2011. Torrejón (2013).

Peniophora lycii (Pers.) Höhn. \& Litsch. [=Peniophora caesia Bres.]

On branch opf Ceratonia siliqua, Lefkara, Jun. 1931. On a decaying branch of Cistus creticus, Asgata II, 24 Nov. 2011. Nattrass (1937); Torrejón (2013). As Peniophora caesia Bres. in the first reference.

Peniophora meridionalis Boidin

On a decaying branch of Quercus infectoria, Agia Paraskevi, 23 Nov. 2011. Torrejón (2013).

Peniophora quercina (Pers.) Cooke

On a decaying branch of Quercus infectoria, Agia Paraskevi, 23 Nov. 2011. Torrejón (2013). 
Peniophora sp.

On Corylus avellana (without date and site). Zyngas (1973 from Georghiou \& Papadopoulos 1957).

Phaeolus schweinitzii (Fr.) Pat. [=Polyporus schweinitzii Fr.]

On trunk of Pinus halepensis, Stavros, Jan. 1936. Nattrass (1937).

Phallus impudicus L.

Under Cistus sp. Karaman, Jan. 2001. Wynei (2005).

Phellinus igniarius (L.) Quél.

On tamarisk, Ortaköy, Jan. 2001. Wynei (2005).

Phellinus pomaceus (Pers.) Maire [=Fomes pomaceus (Pers.) Lloyd]

On trunk of Prunus persica, Pedhoulas, Jun. 1931; on branch of Prunus cerasus, Prodhromos, Oct. 1933; on branch of Prunus amygdalus, Agros, Jan. 1936; on branch of Prunus domestica, Ay. Amvrosios, Kyrenia, Feb. 1936; on trunk and branch of Prunus armeniaca, Limassol, Feb. 1936. Nattrass (1937).

Phellinus rimosus (Berk.) Pilát

On a tree-size terebinth, Karaağaç. Wynei (2005).

Phellodon tomentosus (L.) Banker

Coniferous and mixed forests, Alevkaya, autumn and spring. Momany \& Gücel (2009).

Phoeomana speirea (Fr.) Redhead [=Mycena speirea (Fr.) Gillet]

On wood and twigs, Alevkaya, Mar. Momany \& Gücel (2009).

Pholiota highlandensis (Peck) Quadr. \& Lunghini [=Pholiota carbonaria (Fr.) Singer] On burnt ground, Beşparmak peaks, Dec.-Jan. 1995-1996. On soil surface in burned forests, Alevkaya, autumn. Wynei (2005); Momany \& Gücel (2009). Many groups seen after the 1995 fire.

Pholiota mixta (Fr.) Kuyper \& Tjall.-Beuk.

On buried conifer wood, Buffavento, dirt road, Jan. 2001. Wynei (2005).

Phylloporia ribis (Schumach.) Ryvarden [=Fomes ribis (Schumach.) Gillet]

On collar of Rosa sp., Nicosia, Jul. 1931. Parasitic on a branch of Cistus sp., Kayalar, Dec. 2001. On Rosa sp. (without date and site). Nattrass (1937); Wynei (2005); Zyngas (1973 from Nattrass \& Papaioannou 1938). As Fomes ribis (Schumach.) Gillet in the first reference, and as Phellinus ribis (Schumach.) Quél. in the last one.

Pisolithus arrhizus (Scop.) Rauschert

Under Eucalyptus sp., Vadili (without date). Wynei (2005). Widely scattered elsewhere under other trees.

Pleurotus eryngii (DC.) Quél.

On rich humus on the ground amongst moss, Taşkent, Pigades-Hisarköy, Geçitköy and Tepebasi Altkisivolari, Mar. Momany \& Gücel (2009).

Pleurotus eryngii var. ferulae (Lanzi) Sacc. [=Pleurotus fuscus var. ferulae (Lanzi) Bres.]

Among and probably in association with Ferula communis, Salamis, 1931. Dec.-Apr., (without a specific site data). Growing saprotrophically or parasitically on Ferula communis, (without date). On giant fennel plants or remains, between autumn and spring. Nattrass (1937); Wynei (2005); Loizides (2008); Loizides et al. (2011). A much esteemed comestible and frequently offered for sale. As Pleurotus fuscus var. ferulae (Lanzi) Bres. In the first reference. 
Pleurotus ostreatus (Jacq.) P. Kumm.

On various broadleaved trees (whithout date). Loizides et al. (2011). Rare.

Pleurotus pulmonarius (Fr.) Quél.

On deciduous wood or buried roots, Tepebaşi, Apr. Momany \& Gücel (2009).

Pluteus aff. cinereofuscus

In a damp site, Karaman, Nov. 1998. Wynei (2005).

Polyporus meridionalis (A. David) H. Jahn [=Polyporus meridionalis (A. David) Tellería]

On fallen twigs of deciduous trees, Karaoğlanoğlu, Mar. On fallen Cistus twigs and debris of other sclerophyllous plants, growing throughout the year. Wynei (2005); Momany \& Gücel (2009); Loizides \& Kyriakou (2011). Quite common on roots of pine trees and garigue shrubs. As Polyporus meridionalis (A. David) Tellería in the second reference.

Polysaccum crassipes DC.

Under Eucalyptus sp., Oct. 1932. Nattrass (1937).

Porodaedalea pini (Brot.) Murrill [=Trametes pini (Brot.) Fr.]

On trunk of Pinus halepensis, Kyrenia, Jun. 1931. Nattrass (1937).

Psathyrella artemisiae (Pass.) Konrad \& Maubl. (as Psathyrella squamosa (P. Karst.)

M. M. Moser)

In mixed woodland, Alevkaya, autumn and spring. Momany \& Gücel (2009).

Psathyrella bipellis (Quél.) A. H. Sm.

On turf among Dittrichia sp. Shrublets in a damp valley, Kalkanli, Dec. 1999. Wynei (2005).

Psathyrella candolleana (Fr.) Maire

On turf around pinewoods, Atifonitis monastery, Feb. 2002. Near deciduous trees, stumps or cut trees, Girne-Alsancak and Karaoğlanoğlu, Feb. Wynei (2005); Momany \& Gücel (2009). Rather common.

Psathyrella sp.1.

Several troops on turf in olive valley, Kalkanli, Jan. 2001. Wynei (2005).

Psathyrella sp.2.

In troops on grassy bank, Mounty Palace, Mar. 2001. Wynei (2005).

Psathyrella spadiceogrisea (Schaeff.) Maire

On mixed tree-litter, Alevkaya, Jan. 2001. Wynei (2005).

Psilocybe crobula (Fr.) Singer

On fallen pine-bark, St. Hilarion, Dec. 2000. On wood chips and plant remanins, rarely also on manure, autumn and winter. Wynei (2005); Loizides et al. (2011). Uncommon.

Ramaria formosa (Pers.) Quél.

In black pine forest, under golden oak (without date). Loizides et al. (2011). Rare.

Rectipilus cistophilus Esteve-Rav. \& Vila

On a decaying twig of Cistus creticus, Agia Paraskevi, 23 Nov. 2011. Torrejón (2013).

Rhizopogon luteolus Fr. et Nordholm

Under Cistus spp. Kormakiti forest, (without date). In light soils, (without site and date). In mixed forest especially under coniferous, Alevkaya, autumn. Nattrass (1937); Wynei (2005); Momany \& Gücel (2009). Fairly common.

Rhizopogon roseolus (Corda) Th. [=Rhizopogon vulgaris (Vittad.) M. Lange]

On bare soil, Karraagaç, Nov. 1997. Wynei (2005). 
Rhodocybe gemina (Paulet) Kuyper \& Noordel.

In pinewoods, beside Gecitköy lake and E of Akdeniz, Dec. 2001. Wynei (2005).

Rhodocybe popinalis (Fr.) Singer

On turf among pines, near Ermeni evi below Alevkaya, Jan. 2001. Wynei (2005).

Rigidoporus sanguinolentus (Alb. \& Schwein.) Donk

On decaying wood of Pinus nigra subsp. palliata, Troodos, 17 Nov. 2011. Torrejón (2013).

Rigidoporus ulmarius (Sowerby) Imazeki [=Fomes ulmarius (Sowerby) Fr.]

On trunk of Populus nigra. Ay. Nikolaos, Paphos, 1933. At the trunk base of deciduous trees, usually carob, Alevkaya, Mar. Nattrass (1937); Momany \& Gücel (2009). As Fomes ulmarius (Sowerby) Fr. in the first reference.

Ripartites tricholoma (Alb. \& Schwein.) P. Karst.

On soil under pines, Alevkaya, 1998. Wynei (2005). Occasional.

Rugosomyces carneus (Bull.) Bon [=Calocybe carnea (Bull.) Kühner]

Amongst grass, Alevkaya, winter. Momany \& Gücel (2009).

Rugosomyces chrysenteron (Bull.) Bon

Under Pinus sp., Alrvkaya, Dec. 1998. Wynei (2005).

Rugosomyces onychinus (Fr.) Raithelh.

In pinewoods, Alevkaya, Dec. 1998. Wynei (2005).

Russula acrifolia Romagn.

In black pine forests, early in autumn. Loizides et al. (2011). Common.

Russula aurea Pers.

In black pine forests, early in autumn in high temperatures. Loizides et al. (2011). Frequent.

Russula chloroides (Krombh.) Bres.

Without data. In black and calabrian pine forests, as well as golden oak forests, early in autumn. Loizides (2008); Loizides et al. (2011). Common.

Russula delica Fr.

Without data. On pine-litter, Yayla, Nov. 2000. In all kinds of forests, early in autumn. Wynei (2005); Loizides et al. $(2008,2011)$. One of the commonest species in Cyprus.

Russula insignis Quél.

Associated with Quercus and Cistus, in early autumn. Loizides \& Kyriakou (2011).

Russula luteotacta Rea

Under oak an carob isolated among pines, Antiphonitis, Nov. 1997. In black and calabrian pine forests, as well as alluvial broadleaved forests, autumn. Wynei (2005);

Loizides et al. (2011). Common.

Russula medullata Romagn.

In black pine forests, very early in autumn in high temperatures. Loizides et al. (2011). Frequent.

Russula sanguinea (Bull.) Fr.

In pinewood, Tazik Kiran and Mt. Yayla, Dec. 1998. Amongst grass in mixed forests with mostly coniferous trees, Alevkaya, autumn. Wynei (2005); Momany \& Gücel (2009).

Russula torulosa Bres.

Under pine (without site), Dec. 2004. Wynei (2005). 
Russula spp.

Without data. Loizides (2008).

Sarcodon sp.

Under pine and near Arbutus, opposite the picnic spot on the ridge road to Kantara, Dec. 2004. Wynei (2005).

Schizophyllum commune Fr.

A common saprotroph on dead wood, (without date). On a willow stump, Girne, (without date). On dead wood of deciduous trees and stumps, Alevkaya, Apr. Nattrass (1937); Wynei (2005); Momany \& Gücel (2009).

Schizopora paradoxa (Schrad.) Donk

On dead wood especially deciduous trees, Alevkaya, Apr. Momany \& Gücel (2009).

Scleroderma verrucosum (Bull.) Pers.

On soil beside a concrete step, Karaman, Nov. 1997. In heath, parks and woodland, Alevkaya, autumn. In calabrian pine and alluvial forests, cultivated land and Mediterranean maquis. Wynei (2005); Momany \& Gücel (2009). Loizides et al. (2011). Occasional.

Skeletocutis nivea (Jungh.) Jean Keller

On a dead pine branch, Alevkaya, (without date). Wynei (2005). Common in the woods near Alevkaya.

Sparassis crispa (Wulfen) Fr.

On stumps and roots of various coniferous trees, autumn and winter. Loizides et al. (2011). Rare.

Stereum hirsutum (Willd.) Pers.

A common saprotroph on dead wood, (without date). On olive trunk, (without site and date). On fallen branches and stumps of deciduous trees, Selvilitepe, Apr. Nattrass (1937); Wynei (2005); Momany \& Gücel (2009).

Stereum ochraceoflavum (Schwein.) Sacc.

On wood from decaying branches of Quercus alnifolia, Psilo Dendra, 17 Nov. 2011. Torrejón (2013).

Stropharia aeruginosa (Curtis) Quél.

Found hidden among carobs, Lapta, Dec. 2000. Wynei (2005).

Stropharia coronilla (Bull.) Quél.

Among reed-beds, özhan, Dec. 1998. Among lawns, parks and pasture, Alevkaya, winter. Wynei (2005); Momany \& Gücel (2009).

Suillus luteus (L.) Roussel

In black and calabrian pine forests, autumn and winter. Loizides et al. (2011). Common.

Suillus bellinii (Inzenga) Watling

In pinewoods under isolated carob and oak, Esentepe, Nov. 1999; Jan. 2000. In black and calabrian pine forests, autumn and winter. Wynei (2005); Loizides et al. (2011). Common.

Suillus bovinus (L.) Roussel

Under coniferous trees, at the campus of the Near East University, winter. Momany \& Gücel (2009). 
Suillus collinitus (Fr.) Kuntze [=Suillus fluryi Huijsman]

In mixed woodland forests, Alevkaya, autumn. In black and calabrian pine forests, autumn and winter. Momany \& Gücel (2009); Loizides et al. (2011). Common. As Suillus fluryi Huijsman in the first reference.

Suillus granulatus (L.) Roussel

On pine litter, Alevkaya, Jan. 2001. Mycorrhizal in association with pine roots, at the campus of Near East University; under pine trees, Nicosia, Dec. to Jan. Wynei (2005); Momany \& Gücel (2009).

Suillus luteus (L.) Roussel

Without data. Loizides (2008).

Suillus mediterraneensis Jacquet. \& J. Blum) Redeuilh

On pine litter, Alevkaya, Jan. 2001. Wynei (2005).

Suillus tridentinus (Bres.) Singer

Under coniferous trees, Alevkay, autumn. Momany \& Gücel (2009).

Suillus spp.

Without data. Loizides (2008).

Tapinella panuoides (Batsch) E.-J. Gilbert [=Paxillus panuoides (Fr.) Fr.]

On conifer stumps, Karaman 2000; on ground debris probably attached to hidden roots, Cengizköy 1998. On conifer woods stumps, Viran Kilise, (without date). Wynei (2005); Momany \& Gücel (2009).

Thanatephorus cucumeris (A. B. Frank) Donk [=Corticium solani (Prill. \& Declacr.) Bourdot \& Galzin]

On Dianthus caryophyllus, (without date and site). Zyngas( 1973 from Georghiou \& Papadopoulos 1957).

Thanatephorus sterigmaticus (Bourdot) P. H. B. Talbot

On a non decorticated dead stem of Cistus creticus, Foini, 21 Nov. 2011. Torrejón (2013). Rare.

Thelephora caryophyllea (Schaeff.) Pers.

Under Cistus, autumn, winter and spring. Loizides \& Kyriakou (2011).

Tomentella asperula (P. Karst.) Höhn. \& Litsch.

On a decaying acorn of Quercus alnifolia, Trail to Calydonian Falls, 19 Nov. 2011. Torrejón (2013).

Trametes pubescens (Schumach.) Pilát

On an apple stump, Lefke, Feb. 1997. Wynei (2005).

Trametes versicolor $(\mathrm{L}$.$) Lloyd [=Polystictus versicolor (\mathrm{L}$.$) Fr.], [=Coriolus versicolor$ (L.) Quél.]

On dead wood of Casuarina equisitifolia, Nicosia, Feb. 1935. Nattrass (1937). On a carob stump, Kozam, Dec. 1998. On deciduous wood, Alevkaya, during the hole year. Wynei (2005); Momany \& Gücel (2009). As Coriolus versicolor (L.) Quél. in the second reference.

Tremella mesenterica Retz.

On the cut surface of a pine, Alevkaya, Dec. 2001. Wynei (2005).

Tricholoma albobrunneum (Pers.) P. Kumm.

Under Pinus halepensis, Troodos, Feb. 1932. Nattrass (1937). Occasionally offered for sale in markets. 
Tricholoma caligatum (Viv.) Ricken

Without data. In black and calabrian pine, as well as golden oak forests, autumn and winter. Loizides et al. (2008, 2011). Frequent.

Tricholoma equestre (L.) P. Kumm.

In black and calabrian pine forests, autumn and winter. Loizides et al. (2011). Frequent.

Tricholoma fracticum (Britzelm.) Kreisel

On pine litter, Mt. Yayla (without date). In calabrian pine forests, autumn and winter. Wynei (2005); Loizides et al. (2011). Common.

Tricholoma portentosum (Fr.) Quél.

Deeply buried in pine litter, Kozan, Jan. 2001. Wynei (2005).

Tricholoma spp.

Without data. Loizides (2008).

Tricholoma terreum (Schaeff.) P. Kumm.

In pinewoods, mid-winter, (whithout site). In black and calabrian pine forests, cedar forests, wood edges and mediterranean maquis, autumn and winter. Under pine needles in mixed forests, Karşiyaka, Mar. Wynei (2005); Loizides et al. (2011); Momany \& Gücel (2009). Common.

Tubaria conspersa (Pers.) Fayod

On soil and woody debris in a garden under Oxalis pes-caprae, Karaman Nov. 1999. Wynei (2005).

Tubaria dispersa (L.) Singer [Tubaria autochtona (Berk \& Broome) Sacc.]

On soil, Karaman, Dec. 1998; under Zizyphus bush, perhaps growing on its buried fruits, Kanliköy. Wynei (2005).

Tubaria furfuracea (Pers.) Gillet

In manure grass among Dittichia viscosa, Özhan, Dec. 1998; under Zizyphus, Kalavaç. Wynei (2005).

Tulostoma squamosum Pers.

In turf around the ruined church, Alevkaya, Jan. 1999. Wynei (2005).

Volvariella gloiocephala (DC.) Boekhout \& Enderle

In sheltered grassy place, especially if manure, Alevkaya and Karaman, Dec. 1998. In fields, pastures and road edges. Wynei (2005); Loizides et al. (2011). Frequent.

Vuilleminia comedens (Nees) Maire

On a decaying branch of Quercus alnifolia and on decaying branch of Arbutus andrachne, Psilo Dendra, 17 Nov. 2011. Torrejón (2013).

Vuilleminia macrospora (Bres.) Horstam

On wood from branches of Cistus creticus, Foini, 21 Nov. 2011. Torrejón (2013).

Vuilleminia megalospora Bres.

On a decaying branch of Quercus alnifolia, Trail to Calydonian Falls, 19 Nov. 2011. Torrejón (2013). Rare.

Xerocomellus chrysenteron (Bull.) Šutara [=Xerocomus chrysenteron (Bull.) Quél.]

In black and calabrian pine forests, autumn. Loizides et al. (2011).

Xerocomellus aff. chrysenteron [=Xerocomus aff. chrysenteron]

On the roadside pine litter, Esentepe, Nov. 2000. Wynei (2005).

Xeromphalina cauticinalis (With.) Kühner \& Maire [=Xeromphalina fellea Maire \& Malençon]

Under conifers, Alevkaya, Jan. 1999. Wynei (2005). 


\section{CONCLUSIONS}

At the time the author was looking for references and information dealing with fungi in Cyprus Island; the oldest documented information collected was Chatin (1897). For this reason, it is supposed that the history of the mycology in Cyprus started with this data. Despite the fact that Cyprus Island is reported to be an area with rather scarce surveys and mycological literature, a good bunch of works dealing with fungi in this area is aported in this report. The most important contribution of the current paper is the compiled information broughting together different works dealing with fungi in this area throughout the three centuries of mycology in Cyprus. Several macrofungi labelled as rare in the notes of this checklist such as Amanita torrendii, Chlorophyllum molybdites, Conferticium ochraceum, Hyphoderma nemorale, Myriostoma coliforme, Rectipilus cistophilus, Sparassis crispa, Thanatephorus sterigmaticus, Tomentella asperula and Vuilleminia megalospora, should be considered under protection by local authorities. Nevertheless, Battarrea stevenii and Boletus rhodoxanthus, wich are catalogued in many European countries as endangered species, appear to be very common in Cyprus.

Nowadays, only a small group of local amateur mycologists are dealing with macrofungi in Cyprus, and no one with microfungi. We are far away to know the mycobiota of the island, so it will be very important to organize new forays similar to British Mycological Association's Overseas Meeting 2011 in order to improve the knowledge of fungi in this area.

Acknowledgements. The author would like to express his thanks to Mr Michael Loizides, Dr. Salih Gücel and Dr. Stephanos Diamandis for providing Cypriot bibliography.

\section{REFERENCES}

Altson R.A. 1956. Report on an investigation into the cause of a root-rot of broad beens (Vicia faba) in Cyprus. Department of Agriculture. The Government of Cyprus, Nicosia, Cyprus.

Chatin M.A. 1897. Un Nouveau Terfas (Terfezia aphroditis) de Lille de Chypre. Bulletin de la Société Botanique de France 44: 290.

Georghiou G.P., Papadopoullos C. 1957. A second list of Cyprus fungi. Department of Agriculture. The Government of Cyprus, Nicosia, Cyprus.

Loizides M. 2008. A secret world: The fungi of Cyprus. Field Mycology 9 (3): 107-109.

Loizides M., Kyriakou T. 2011. Fungi of Cistus Maquis. Field Mycology 12 (1): 14-22.

Loizides M., Kyriakou T., Tziakouris A. 2011. Edible and Toxic Fungi of Cyprus. Manitari, Greece.

Loizides M., Hobart C., Konstandinides G., Yiangou Y. 2012. Desert Truffles: The mysterious jewels of antiquity. Field Mycology 13 (1): 17-21.

Momany A., Gücel S. 2009. Mushrooms of north Cyprus, ecology, distribution, classification and toxicity. Near East University, Nicosia, North Cyprus.

Momany A.T., Mohamad A., Gücel S. 2009. A comprehensive Study on Agaricus species of north Cyprus. World Journal of Agricultural Sciences 5 (2): 195-200.

Nattrass R.M. 1937. A first list of Cyprus Fungi. Department of Agriculture, The Government of Cyprus, Nicosia, Cyprus.

Nattrass R.M., Papaioannou P. 1938. Aditions to "A first list of Cyprus fungi”. Department of Agriculture, The Government of Cyprus, Nicosia, Cyprus.

Neophytou G., Ioannou N. 2009. First report of false smut disease on date palms in Cyprus. Journal of Plant Pathology 91 (1): 240. 
Torrejón M. 2013. Fungi of Cyprus: new data on micro- and macrofungi. Acta Mycol. 48 (2): 207-218.

Tsopelas P., Nikolau K. 2005. First report of Heterobasidion annosum in Cyprus. Plant Pathol. 54 (4): 583. DOI: $10.1111 / \mathrm{j} .1365-3059.2005 .01202 . \mathrm{x}$

Tsopelas P., Angelopoulos A., Nikolau K. 2008. Seiridium cardinale is a new threat to cypress trees in Cyprus. Plant Pathol. 57 (4): 784. DOI: 10.1111/j.1365-3059.2007.01812.x

Willoughby L.G. 1983. The bacterial antagonist of Karlingia rosea; further observations from Spain and Cyprus. Nova Hedwigia 38: 113-128.

Winey D.E. 2005. An illustrated introduction to the larger fungi of north Cyprus. Richmond Publishing Company, UK.

Zyngas J.P. 1973. The Cyprus fungi. Department of Agriculture, Ministry of Agriculture and Natural Resources, Nicosia, Cyprus. 